\title{
Gastric bypass and banding equally improve insulin sensitivity and $\beta$ cell function
}

\author{
David Bradley, ${ }^{1}$ Caterina Conte, ${ }^{1,2}$ Bettina Mittendorfer, ${ }^{1} \mathrm{~J}$. Christopher Eagon, ${ }^{1} \mathrm{~J}$. Esteban Varela, ${ }^{1}$ \\ Elisa Fabbrini, ${ }^{1}$ Amalia Gastaldelli, ${ }^{3}$ Kari T. Chambers, ${ }^{1}$ Xiong Su, ${ }^{1}$ Adewole Okunade, ${ }^{1}$ \\ Bruce W. Patterson, ${ }^{1}$ and Samuel Klein ${ }^{1}$
}

\begin{abstract}
${ }^{1}$ Center for Human Nutrition, Washington University School of Medicine, St. Louis, Missouri, USA. ${ }^{2 D e p a r t m e n t ~ o f ~ C l i n i c a l ~ M e d i c i n e, ~}$ Sapienza University of Rome, Rome, Italy. ${ }^{3}$ Institute of Clinical Physiology of the National Research Council, Pisa, Italy.
\end{abstract}

\begin{abstract}
Bariatric surgery in obese patients is a highly effective method of preventing or resolving type 2 diabetes mellitus (T2DM); however, the remission rate is not the same among different surgical procedures. We compared the effects of $20 \%$ weight loss induced by laparoscopic adjustable gastric banding (LAGB) or Roux-en-Y gastric bypass (RYGB) surgery on the metabolic response to a mixed meal, insulin sensitivity, and $\beta$ cell function in nondiabetic obese adults. The metabolic response to meal ingestion was markedly different after RYGB than after LAGB surgery, manifested by rapid delivery of ingested glucose into the systemic circulation, by an increase in the dynamic insulin secretion rate, and by large, early postprandial increases in plasma glucose, insulin, and glucagon-like peptide-1 concentrations in the RYGB group. However, the improvement in oral glucose tolerance, insulin sensitivity, and overall $\beta$ cell function after weight loss were not different between surgical groups. Additionally, both surgical procedures resulted in a similar decrease in adipose tissue markers of inflammation. We conclude that marked weight loss itself is primarily responsible for the therapeutic effects of RYGB and LAGB on insulin sensitivity, $\beta$ cell function, and oral glucose tolerance in nondiabetic obese adults.
\end{abstract}

\section{Introduction}

Obesity is an important cause of insulin resistance and impaired pancreatic $\beta$ cell function, which leads to the development of type 2 diabetes mellitus (T2DM) (1). Weight loss in obese people has potent beneficial metabolic effects and can improve both multiorgan insulin sensitivity $(2,3)$ and $\beta$ cell function $(4,5)$. In fact, in most patients, marked weight loss induced by bariatric surgery results in complete resolution of T2DM, usually defined as discontinuation of all diabetes medications in conjunction with some evidence of normal glycemic control (fasting blood glucose concentration or glycated hemoglobin) (6). However, the remission rate is not the same among surgical procedures. Procedures that divert ingested nutrients from passage through the upper gastrointestinal tract, such as Roux-en-Y gastric bypass (RYGB) surgery, have much higher remission rates than do procedures that simply restrict the stomach, such as laparoscopic adjustable gastric banding (LAGB) surgery (6). This observation has led to the notion that anatomical diversion of the upper gastrointestinal tract has important therapeutic effects on glucose homeostasis that are independent of weight loss itself. However, the interpretation of this clinical observation is confounded by differences in weight loss among surgical treatment groups (6).

The main purpose of this study was to test the hypothesis that upper gastrointestinal tract diversion has weight loss-independent therapeutic effects on the 2 major factors involved in the pathogenesis of T2DM: $\beta$ cell function and insulin action. We evaluated the metabolic response (glucose kinetics and $\beta$ cell function) to a mixed meal, using stable isotopically labeled tracer methods in conjunction with mathematical modeling, and assessed insulin sensitivity,

Conflict of interest: Samuel Klein serves on a Scientific Advisory Board for Ethicon Endo-Surgery.

Citation for this article: J Clin Invest. 2012;122(12):4667-4674. doi:10.1172/JCI64895. using the hyperinsulinemic-euglycemic clamp procedure and stable isotopically labeled tracer infusion, in nondiabetic obese subjects both before undergoing RYGB or LAGB surgery and after surgeryinduced loss of a targeted $20 \%$ of body weight. We also evaluated the effects of surgery-induced weight loss on cellular factors purported to be involved in regulating metabolic function, specifically adipose tissue inflammatory factors (gene expression of macrophage markers, chemokines, and cytokines) (7) and intramyocellular lipid intermediates (diacylglycerol [DAG] and ceramide content) (8).

\section{Results}

\section{Body composition and basal metabolic variables}

Subjects in the LAGB and RYGB groups lost $19.3 \% \pm 1.9 \%$ and $20.1 \% \pm 2.3 \%$ of their body weight by $22 \pm 7$ and $16 \pm 2$ weeks after surgery, respectively (Table 1 ). The delay in achieving the target weight loss in the LAGB group was primarily due to a slower rate of weight loss in the first 6 weeks after surgery, before the first band adjustment was performed. Weight loss caused marked changes in body composition, but the effects on fat-free mass (FFM), fat mass (FM), intra-abdominal adipose tissue (IAAT) volume, intrahepatic triglyceride (IHTG) content, and plasma leptin concentration were not different between groups (Table 1). Weight loss also caused beneficial changes in plasma markers of glucose homeostasis (glucose, insulin, C-peptide, and adiponectin concentrations) and inflammation (C-reactive protein [CRP]), but these outcome variables decreased equally in both groups after surgeryinduced weight loss (Table 1).

\section{Insulin sensitivity}

Values for insulin sensitivity, assessed by using the homeostasis model assessment of insulin resistance (HOMA-IR), decreased by more than half after weight loss in the LAGB and RYGB groups, but 


\section{Table 1}

Body composition and metabolic variables before and after LAGB and RYGB surgery-induced weight loss

\begin{tabular}{|c|c|c|c|c|}
\hline & \multicolumn{2}{|c|}{ LAGB } & \multicolumn{2}{|c|}{ RYGB } \\
\hline & Before & After & Before & After \\
\hline Body weight (kg) & $128.7 \pm 29.6$ & $104.1 \pm 24.5^{\mathrm{A}}$ & $132.0 \pm 28.4$ & $105.5 \pm 23.1^{A}$ \\
\hline $\mathrm{BMI}\left(\mathrm{kg} / \mathrm{m}^{2}\right)$ & $46.5 \pm 8.8$ & $37.6 \pm 7.3^{A}$ & $45.6 \pm 6.7$ & $36.4 \pm 5.0^{\mathrm{A}}$ \\
\hline FFM $(\mathrm{kg})$ & $62.5 \pm 13.0$ & $56.8 \pm 11.7^{\mathrm{A}}$ & $62.6 \pm 13.5$ & $56.2 \pm 11.6^{\mathrm{A}}$ \\
\hline FM (kg) & $67.1 \pm 22.5$ & $49.8 \pm 20.1^{\mathrm{A}}$ & $68.2 \pm 17.1$ & $49.4 \pm 12.8^{\mathrm{A}}$ \\
\hline FM (\% body weight) & $51.2 \pm 5.7$ & $45.7 \pm 7.2^{\mathrm{A}}$ & $51.9 \pm 4.1$ & $46.5 \pm 4.0^{A}$ \\
\hline IAAT volume $\left(\mathrm{cm}^{3}\right)$ & $1,587 \pm 440$ & $1,056 \pm 332^{A}$ & $1,912 \pm 917$ & $1,060 \pm 435 \mathrm{~A}$ \\
\hline IHTG content $(\%)$ & $12.8 \pm 9.8$ & $4.6 \pm 4.3^{\mathrm{A}}$ & $10.5 \pm 7.6$ & $3.8 \pm 4.1^{\mathrm{A}}$ \\
\hline Plasma glucose (mmol/l) & $5.3 \pm 0.4$ & $4.7 \pm 0.3^{\mathrm{A}}$ & $5.0 \pm 0.5$ & $4.6 \pm 0.4^{A}$ \\
\hline Plasma insulin $(\mu \mathrm{U} / \mathrm{ml})$ & $12.6 \pm 4.1$ & $6.1 \pm 1.9^{A}$ & $14.5 \pm 7.5$ & $5.7 \pm 2.6^{\mathrm{A}}$ \\
\hline Plasma C-peptide (pmol/l) & $1,130 \pm 262$ & $726 \pm 205^{A}$ & $1,170 \pm 525$ & $699 \pm 205^{A}$ \\
\hline HOMA-IR score & $5.8 \pm 1.9$ & $2.3 \pm 0.8^{A}$ & $5.8 \pm 2.3$ & $1.8 \pm 0.9^{A}$ \\
\hline Glucose AUC $\left(\mathrm{mg} / \mathrm{dl} \times 360 \mathrm{~min} \times 10^{3}\right)$ & $40.3 \pm 2.3$ & $38.0 \pm 2.3^{A}$ & $40.3 \pm 2.7$ & $39.3 \pm 1.3^{\mathrm{A}}$ \\
\hline C-peptide AUC $\left(\mathrm{pmol} / / \mathrm{l} \times 360 \mathrm{~min} \times 10^{3}\right)$ & $764 \pm 108$ & $608 \pm 126^{A}$ & $802 \pm 221$ & $631 \pm 117^{A}$ \\
\hline Insulin AUC $\left(\mu \mathrm{U} / \mathrm{ml} \times 360 \mathrm{~min} \times 10^{3}\right)$ & $11.6 \pm 4.8$ & $6.5 \pm 2.4^{\mathrm{A}}$ & $12.9 \pm 5.4$ & $8.5 \pm 2.4^{A}$ \\
\hline Leptin (ng/ml) & $62.5 \pm 25.6$ & $30.9 \pm 20.4^{A}$ & $64.3 \pm 24.6$ & $23.5 \pm 13.5^{\mathrm{A}}$ \\
\hline Adiponectin $(\mu \mathrm{g} / \mathrm{ml})$ & $8.2 \pm 3.3$ & $11.3 \pm 5.1^{\mathrm{A}}$ & $7.2 \pm 3.2$ & $10.3 \pm 5.1^{A}$ \\
\hline C-reactive protein (ng/l) & $12.4 \pm 9.8$ & $7.2 \pm 5.7^{A}$ & $11.4 \pm 14.3$ & $5.2 \pm 4.8^{A}$ \\
\hline
\end{tabular}

Values are means $\pm S D$. ${ }^{A} P<0.05$ vs. before surgery. No significant effect of group or time-group interaction was observed.

there was no significant difference between groups (Table 1). Insulinmediated stimulation of glucose disposal above basal values nearly doubled after weight loss in both the LAGB and RYGB groups, but there was no significant difference between groups (Figure 1).

\section{$\beta$ Cell function}

Total insulin secretion rate (ISR) in response to the mixed meal decreased after both LAGB and RYGB surgery-induced weight loss, and the decrease was similar in both groups (Figure 2A). Dynamic ISR increased after RYGB-induced, but not LAGB-induced, weight loss (Figure 2B). Static ISR decreased to a similar extent after both LAGB (from $41.3 \pm 4.6 \times 10^{3}$ to $31.5 \pm 5.1 \times 10^{3} \mathrm{nmol} / 1$ per 360 $\mathrm{min}$ ) and RYGB (from $45.1 \pm 13.4 \times 10^{3}$ to $29.2 \pm 5.0 \times 10^{3} \mathrm{nmol} / 1$ per $360 \mathrm{~min}$ ) surgery-induced weight loss. Total $\beta$ cell sensitivity ( $\left.\Phi_{\text {total }}\right)$ decreased after surgery-induced weight loss to the same extent in both groups (Figure 2C). The disposition index (DI) nearly doubled after LAGB- and RYGB-induced weight loss, but there was no difference between groups (Figure 2D).

\section{Metabolic response to a mixed meal}

Plasma hormone concentrations. Postprandial AUCs for plasma insulin and C-peptide concentrations decreased to a similar extent in both groups after surgery-induced weight loss (Table 1). However, RYGB surgery altered the shape of the concentration curves, manifested by a much higher peak in the early rise of plasma insulin and C-peptide after surgery than before (Figure 3 ). In addition, RYGB surgery, but not LAGB surgery, caused a marked increase in postprandial plasma active glucagon-like peptide-1 (GLP-1) concentration (Figure 3). Neither LAGB nor RYGB surgery affected plasma glucagon concentrations (Figure 3).

Glucose kinetics. The AUC for plasma glucose after ingesting the mixed meal decreased to a similar extent in both groups after surgery-induced weight loss (Table 1). However, in contrast to LAGB, RYGB resulted in a much higher peak in the early rise of plasma glucose concentration, because of a marked increase in the early rate of appearance $(\mathrm{Ra})$ of ingested glucose into the systemic circulation (Figure 4). Most of the ingested glucose appeared in the circulation within 120 minutes of initiating meal consumption; the percentage of total meal-derived glucose that appeared in the circulation within 120 minutes of initiating meal ingestion was $75 \% \pm 14 \%$ before and $73 \% \pm 13 \%$ after surgery in the LAGB group, but increased from $70 \% \pm 19 \%$ before to $92 \% \pm 2 \%$ after surgery in the RYGB group $(P=0.01)$. Before surgery, meal ingestion caused a prompt and nearly complete suppression of endogenous glucose production (EGP), which did not return to baseline even 5.5 hours after completing meal consumption (Figure 4). Therefore, EGP accounted for less than $10 \%$, whereas ingested glucose accounted for more than $90 \%$, of glucose in plasma at the end of meal ingestion (Figure 4). The suppression of EGP in response to meal ingestion was not different before and after surgery in the LAGB group. In contrast, RYGB surgery resulted in a more rapid and almost complete suppression of EGP, which rapidly returned to baseline levels.

\section{Cellular factors involved in metabolic regulation}

Subcutaneous adipose tissue inflammation. Weight loss caused a marked decrease in adipose tissue gene expression (as assessed by mRNA content) of proinflammatory macrophage cell surface markers (EMR1 and $C D 11 B)$, chemokines (CCL2), and proinflammatory cytokines (CSF3, IL6, TNFA, and LEP), but increased the expression of the antiinflammatory cytokine IL10 (Figure 5). However, the changes in these pro- and antiinflammatory markers were not different between surgical groups.

Skeletal muscle lipid metabolites. Weight loss did not result in a decrease in either intramyocellular total DAG or ceramide content (Figure 6).

\section{Discussion}

In the present study, we evaluated whether bariatric surgery that bypasses the upper gastrointestinal tract has important weight loss-independent effects on plasma glucose homeostasis in nondiabetic obese subjects after they have experienced considerable sur- 


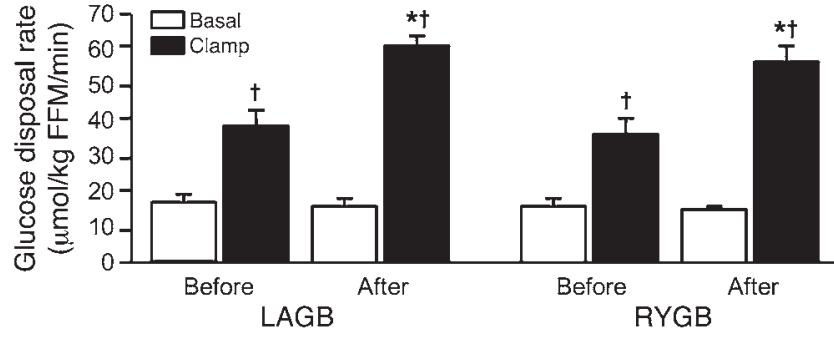

Figure 1

Glucose disposal during basal conditions and insulin infusion before and after $20 \%$ weight loss induced by LAGB or RYGB surgery. ${ }^{\star} P<0.001$ vs. before surgery; ${ }^{t} P<0.001$ vs. basal. Values are means \pm SEM.

gery-induced weight loss. Our data demonstrated that both insulin sensitivity, assessed as insulin-mediated glucose disposal, and $\beta$ cell function, assessed as the DI ( $\beta$ cell response to a mixed meal in relationship to insulin sensitivity), improved markedly after $20 \%$ weight loss, but there were no differences between surgical groups. However, RYGB surgery altered the metabolic response to ingesting a mixed meal, characterized by rapid delivery of ingested glucose into the systemic circulation, which was responsible for a large early increase in plasma glucose concentration and insulin secretion. In contrast, the rate of delivery of ingested glucose into the circulation and the shapes of the plasma glucose and insulin response curves to a mixed meal did not change after LAGBinduced weight loss. These results demonstrated that even though upper gastrointestinal tract diversion caused profound changes in certain aspects of the metabolic response to a meal, weight loss itself was primarily responsible for the long-term therapeutic effects of RYGB and LAGB on $\beta$ cell function, insulin sensitivity, and oral glucose tolerance in nondiabetic obese adults.

Insulin-resistant glucose metabolism in skeletal muscle, one of the most common metabolic abnormalities associated with obesity, is an important risk factor for developing the metabolic syndrome, T2DM, and coronary heart disease (9). Data from previous studies have found that moderate weight loss (5\%-10\%), induced by RYGB surgery or diet therapy alone, does not affect insulin-mediated glucose disposal (10-13). Therefore, the results from our study, in conjunction with the data obtained from these previous studies, indicate that marked weight loss (20\%) is needed to improve skeletal muscle insulin sensitivity in extremely obese people. Moreover, the improvement in insulin sensitivity was the same in both the RYGB and LAGB groups, which demonstrated that RYGB did not have weight loss-independent effects on skeletal muscle insulin action.

Weight loss resulted in a $25 \%$ decrease in total mealinduced insulin secretion in both LAGB and RYGB groups. This decrease was expected, because less insulin is needed to respond to an oral glucose challenge when insulin sensitivity has increased. However, the decline in insulin secretion was much less than the nearly 2 -fold increase in insulin sensitivity. Accordingly, $\beta$ cell function, assessed as total meal-induced insulin secretion in relationship to insulin sensitivity (i.e., DI), increased approximately $75 \%$ in both surgical groups. These data suggest the deterioration in $\beta$ cell

\section{Figure 2}

function that occurs as obese people progress from normal glucose homeostasis to T2DM (14) can be prevented by weight loss induced by either LAGB or RYGB surgery.

The pattern of the metabolic response to mixed meal ingestion was very different after RYGB than after LAGB surgery-induced weight loss. Although total postprandial ISR and plasma glucose concentration AUCs were the same after both surgical procedures, the dynamic ISR, which represents the insulin response to increasing plasma glucose concentration (predominantly during the first 60 minutes after initiating meal ingestion and usually accounting for up to $10 \%$ of total ISR), increased markedly after RYGB surgery, but did not change after LAGB surgery. The increase in the dynamic ISR was likely a direct consequence of the rapid rate of glucose absorption, presumably due to rapid emptying of the meal into the small intestine (15), and subsequent rapid rise in plasma glucose concentration, in conjunction with an increase in the insulin response to plasma glucose because of an increase in plasma GLP-1 $(16,17)$. However, the increase in early meal-induced ISR after RYGB surgery did not prevent a marked increase in the early peak in plasma glucose concentration. The clinical implications of this altered metabolic response to a meal are not clear, but it does not necessarily demonstrate a beneficial effect, because a high peak postprandial glucose concentration is associated with insulin resistance and cardiovascular disease (18-21).

The delivery of ingested glucose into the systemic circulation was responsible for the increase in postprandial plasma glucose concentration, because hepatic glucose production was almost completely suppressed by the meal-induced increase in circulating insulin. This synergy between glucose absorption and EGP is critical to prevent excessive postprandial plasma glucose concentrations. Subjects who had RYGB surgery displayed a more rapid return toward baseline EGP rate than those who had LAGB, thereby preventing a continued decline in plasma glucose in the former group. The increase in EGP in the RYGB group was likely caused by a rapid decline in plasma insulin. These results underscore the importance of hepatic function in preventing postprandial hypoglycemia in patients who have had RYGB surgery.
A

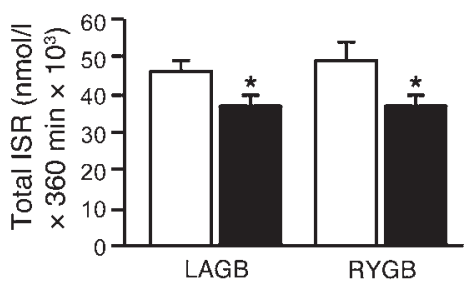

C

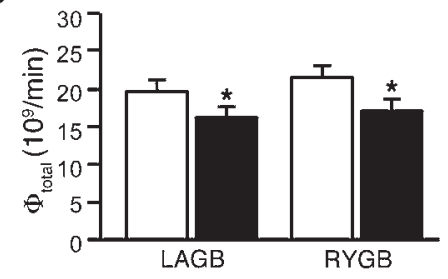

B

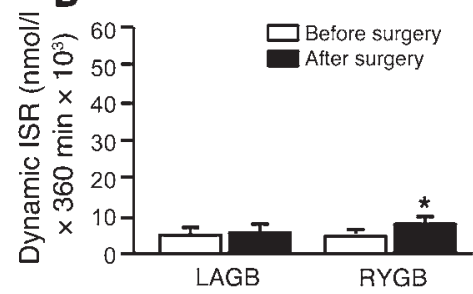

D

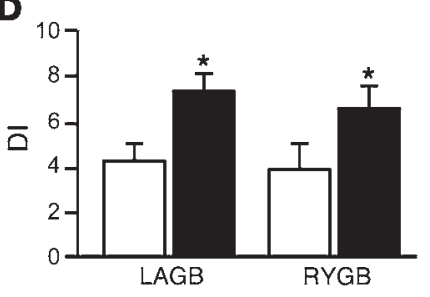

Total ISR (A), dynamic ISR (B), $\Phi_{\text {total }}(\mathbf{C})$, and DI (D) before and after $20 \%$ weight loss induced by LAGB or RYGB surgery. ${ }^{*} P<0.001$ vs. before surgery. Values are means \pm SEM. 

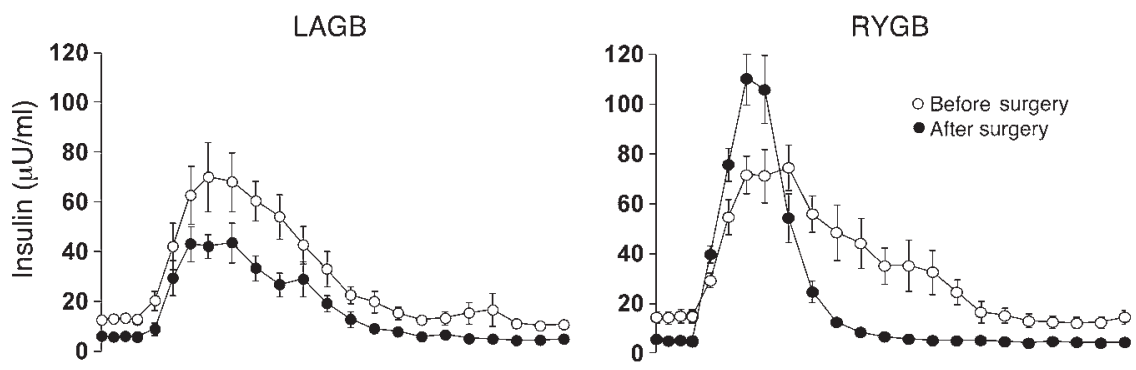

\section{Figure 3}

Plasma hormone concentrations after ingestion of a mixed meal (consumed over 30 minutes) before and after $20 \%$ weight loss induced by LAGB or RYGB surgery. Weight loss-induced changes in plasma insulin, C-peptide, and active GLP-1 concentrations were significantly different between LAGB and RYGB groups $(P<0.0001$ for all). There was no significant effect of weight loss on plasma glucagon concentrations. Values are
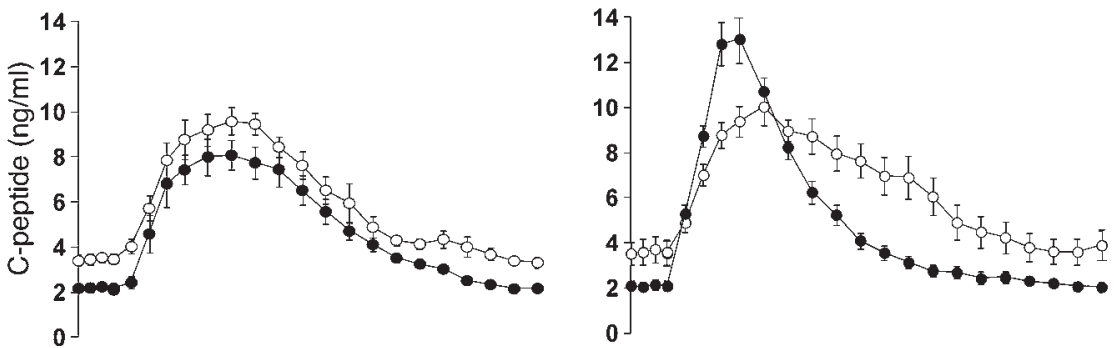
means \pm SEM.
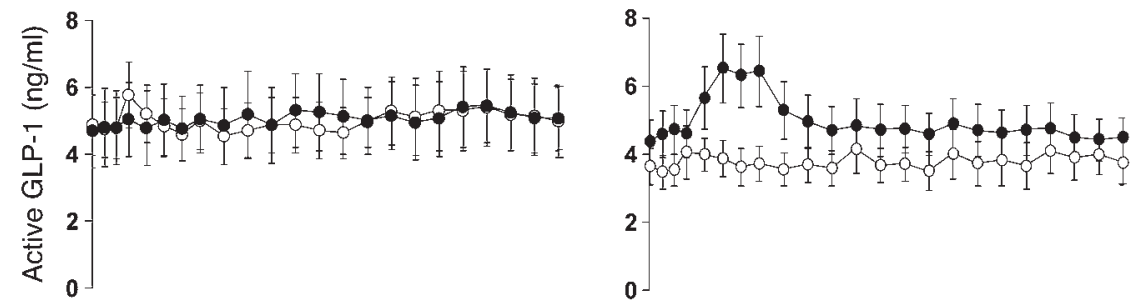

mediators of the improvement in skeletal muscle insulin sensitivity observed in our subjects. These data underscore the need for additional studies to evaluate the importance of intramyocelluar DAG and ceramide in the pathogenesis of skeletal muscle insulin resistance in obese people.

All study subjects were insulin resistant, based on a HOMA-IR value greater than 2.5 , but none had T2DM. Studying subjects without T2DM allowed us to avoid the confounding effects of differences in baseline glycemic control, glucose toxicity, and changes in diabetes medications on our metabolic outcome measures. Accordingly, it cannot be assumed that our results apply to the diabetic population, and additional studies are needed in participants with T2DM. Nonetheless, our study population does not diminish the relevance of our findings, because most patients who undergo bariatric surgery ( $\sim 80 \%)$ do not have T2DM (25).

In conclusion, the results from our study provide insights into the physiological effects of marked RYGB- and LAGB-induced weight loss on the meta-

Our data demonstrated that weight loss, induced by either LAGB or RYGB surgery, had the same beneficial effects on several factors that have been previously proposed to regulate metabolic function in obese people, namely body composition, circulating adiponectin, and markers of inflammation in both plasma and adipose tissue (22). Surgery-induced weight loss decreased body FM, IAAT volume, and IHTG content; increased plasma adiponectin concentration; decreased plasma CRP concentration; and had beneficial effects on multiple pathways of adipose tissue inflammation, manifested by decreased gene expression of proinflammatory macrophages (EMR1 and CD11B), chemokines (CCL2), and cytokines (CSF3, IL6, TNFA, and LEP) and increased gene expression of the antiinflammatory cytokine IL10. However, no differences in any of these outcome measures were detected between surgical groups, which suggests that weight loss itself, rather than the type of surgery, is responsible for the beneficial effects. In contrast, we found that weight loss did not affect intramyocellular DAG or ceramide content, which have previously been shown to impair muscle insulin signaling in rodent models $(23,24)$. Therefore, our results suggest these lipid intermediates were not important bolic response to a meal, on $\beta$ cell function, and on insulin sensitivity in weight loss-matched groups of obese people who do not have T2DM. Our data demonstrated potent but similar beneficial effects of marked weight loss, induced by either LAGB or RYGB, on both $\beta$ cell function and insulin sensitivity in obese people. In contrast, specific aspects of the metabolic response to a mixed meal were profoundly different between surgical procedures, because of rapid absorption of ingested glucose after RYGB that resulted in a marked transient increase in plasma glucose, insulin, and GLP-1 concentrations. However, it is not clear that the alterations in hormone secretion after RYGB have important therapeutic effects on glycemic control, because they did not prevent a large early increase in plasma glucose concentration or result in a greater reduction in postprandial glucose concentration AUC in this group. Therefore, our data do not support the notion of clinically meaningful weight loss-independent effects of RYGB surgery on $\beta$ cell function and insulin sensitivity in nondiabetic obese subjects after they have experienced considerable weight loss. Additional studies are needed to determine whether these findings also apply to obese people with T2DM. 

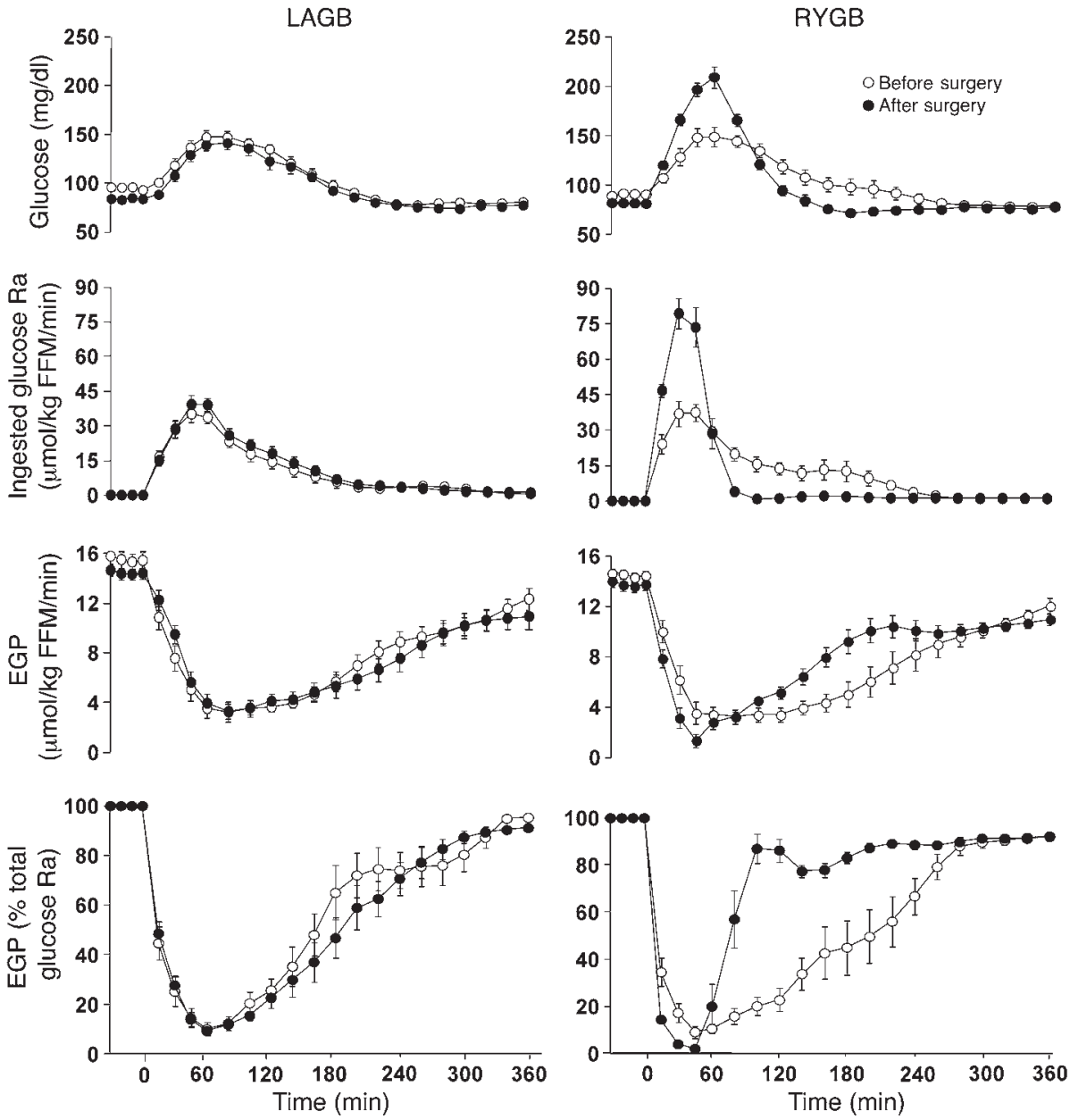

\section{Figure 4}

Plasma glucose concentration, ingested glucose $\mathrm{Ra}$ into the systemic circulation, EGP rate, and EGP as percentage of total glucose $\mathrm{Ra}$ into the systemic circulation after ingestion of a mixed meal (consumed over 30 minutes) before and after $20 \%$ weight loss induced by LAGB or RYGB surgery. Weight loss-induced changes in all parameters shown were significantly different between LAGB and RYGB groups $(P<0.0001)$. Values are means \pm SEM.

\section{Methods}

\section{Selection and description of participants}

20 consecutive, eligible patients who were scheduled to undergo RYGB $(n=10$; 2 men, 8 women; $43 \pm 7$ years old) or LAGB ( $n=10 ; 1$ man, 9 women; $47 \pm 14$ years old) procedures at Barnes-Jewish Hospital (St. Louis, Missouri, USA) participated in this study, which was approved by the Washington University Institutional Review Board. All participants provided written informed consent and completed a comprehensive medical evaluation. Potential participants who smoked cigarettes, had previous malabsorptive or restrictive intestinal surgery, had a history of inflammatory intestinal disease, had evidence of severe organ dysfunction, had uncontrolled hypertension, or had a serum triglyceride concentration of $400 \mathrm{mg} / \mathrm{dl}$ or higher were excluded. Potential participants who had diabetes were excluded to avoid the confounding effects of differences in baseline glycemic control, glucose toxicity, and postsurgical changes in diabetes medications on our outcome measures.

\section{Study design and experimental procedures}

Each subject completed body composition analyses, a hyperinsulinemiceuglycemic clamp procedure, and a mixed-meal metabolic study before bariatric surgery and after $20 \%$ surgery-induced weight loss.

Body composition assessments. Body FM and FFM, IAAT volume, and IHTG content were determined using dual-energy X-ray absorptiometry, magnetic resonance imaging, and magnetic resonance spectroscopy, respectively $(26,27)$.
Hyperinsulinemic-englycemic clamp procedure. Subjects were admitted to the Washington University School of Medicine Clinical Research Unit (CRU) and consumed a standard evening meal ( $12 \mathrm{kcal} / \mathrm{kg}$ FFM; $50 \%$ of calories as carbohydrate, $30 \%$ as fat, $20 \%$ as protein). The following morning, a catheter was inserted into a forearm vein for infusion, and a second catheter was inserted into a radial artery to obtain blood samples. At 6:00 am, a primed, continuous infusion of $\left[6,6-{ }^{2} \mathrm{H}_{2}\right]$ glucose (priming dose, $22 \mu \mathrm{mol} /$ $\mathrm{kg}$; infusion rate, $0.22 \mu \mathrm{mol} / \mathrm{kg} / \mathrm{min}$ ) was started and maintained until the end of the study. At 9:30 am, insulin was infused at a rate of $50 \mathrm{mU} \times \mathrm{m}^{2}$ body surface area $\times \min ^{-1}$ for 4 hours. Euglycemia (plasma glucose $\sim 100$ $\mathrm{mg} / \mathrm{dl}$ ) was maintained by infusion of $20 \%$ dextrose enriched to $2.5 \%$ with $\left[6,6-{ }^{2} \mathrm{H}_{2}\right]$ glucose. Blood samples were obtained immediately before starting the tracer infusion and during the final 30 minutes of the basal period and the clamp procedure to determine plasma substrate and insulin concentrations as well as glucose tracer/tracee ratios (TTRs).

Subcutaneous abdominal adipose tissue and vastus lateralis muscle tissue samples were obtained by percutaneous biopsy 60 minutes after starting the glucose tracer infusion during basal conditions. Biopsy sites were cleaned and draped. After anesthetizing the skin and underlying tissues with lidocaine, adipose tissue was aspirated from the periumbilical area using a 14-gauge needle, and skeletal muscle was obtained using Tilley-Henkel forceps (Sontec Instruments). Tissue samples were immediately rinsed with ice-cold saline and frozen in liquid nitrogen before being stored at $-80^{\circ} \mathrm{C}$.

Mixed meal metabolic study. Subjects were admitted to the CRU and consumed a standard evening meal. The following morning, a catheter 

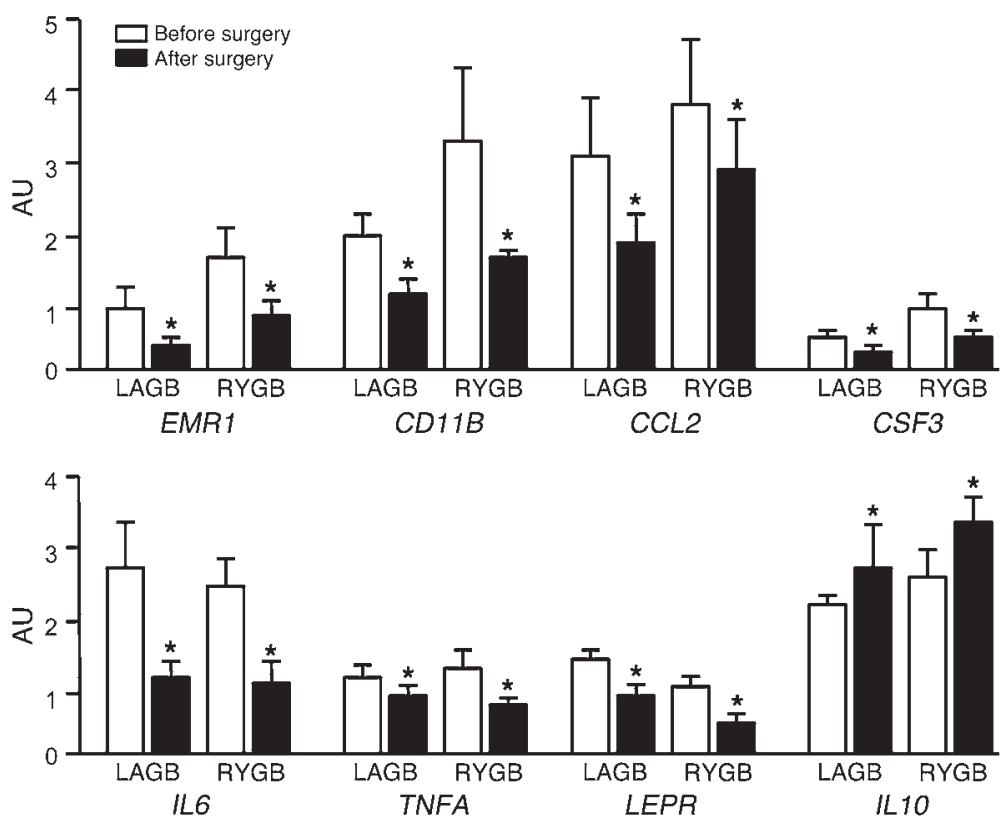

\section{Figure 5}

Adipose tissue gene expression of proinflammatory macrophage cell surface markers (EMR1 and CD11B), chemokines (CCL2), cytokines (CSF3, IL6, TNFA, and $\angle E P)$, and the antiinflammatory cytokine $I L 10$ before and after $20 \%$ weight loss induced by LAGB $(n=8)$ or RYGB $(n=8)$ surgery. ${ }^{*} P<0.05$ vs. before surgery. Values are means \pm SEM was inserted into a forearm vein for infusion, and a second catheter was inserted into a radial artery to obtain blood samples. A primed, continuous infusion of [6,6- $\left.{ }^{2} \mathrm{H}_{2}\right]$ glucose (priming dose, $22 \mu \mathrm{mol} / \mathrm{kg}$; infusion rate, $0.22 \mu \mathrm{mol} / \mathrm{kg} / \mathrm{min}$ ) was started and continued until the end of the study. After 3.5 hours of tracer infusion, subjects ingested a liquid meal (containing $46 \mathrm{~g}$ glucose mixed with $0.9 \mathrm{~g}\left[\mathrm{U}-{ }^{13} \mathrm{C}\right]$ glucose, $9 \mathrm{~g}$ fat, and $9 \mathrm{~g}$ protein), which was provided in 7 equally divided aliquots given every 5 minutes over a 30-minute period. Blood samples were obtained immediately before starting the glucose tracer infusion, every 10 minutes for 30 minutes just before starting the meal ingestion, and then every 15 minutes for the first hour and every 20 minutes for the subsequent 5 hours after starting the meal, to determine plasma substrate and hormone concentrations as well as glucose TTRs.

\section{Surgical procedures}

Bariatric surgeries were performed using standard laparoscopic approaches. The RYGB procedure involved creating a small $(\sim 30 \mathrm{ml})$ proximal gastric pouch and a stapled gastrojejunostomy. A $100-\mathrm{cm}$ Roux limb was constructed by transecting the jejunum $30 \mathrm{~cm}$ distal to the ligament of Treitz and performing a stapled jejunojejunostomy at this site (28). The standard pars flaccid technique was used for LAGB (Lap-Band; Allergan; ref. 29).

\section{Weight management after surgery}

Subjects participated in a supervised dietary weight loss program to help subjects in both groups consume a similar energy-deficit diet and achieve a $20 \%$ weight loss within 4-6 months after surgery. All subjects were instructed to consume a clear liquid diet $(400-600 \mathrm{kcal} / \mathrm{d})$ for the first 2 days after surgery, a full liquid diet (400-600 $\mathrm{kcal} / \mathrm{d})$ on postoperative day 3 , a pureed diet $(2-3 \mathrm{oz} /$ meal providing $700-800 \mathrm{kcal} / \mathrm{d}$ ) on days $4-7$, and a soft diet (3-4 oz/meal providing $800-1,000 \mathrm{kcal} / \mathrm{d}$ ) on days $8-29$, followed by a regular-food diet containing 1,000 $1,200 \mathrm{kcal} / \mathrm{d}$ and $1.0 \mathrm{~g}$ protein $/ \mathrm{kg}$ body weight $/ \mathrm{d}$. After subjects achieved a $20 \%$ weight loss, a balanced weight maintenance diet was prescribed, and subjects main-

\section{Figure 6}

tained a stable body weight ( $<2 \%$ change) for at least 2 weeks before repeat studies were performed.

\section{Analyses of blood samples}

Plasma glucose concentration was determined using an automated glucose analyzer (YSI 2300 STAT plus; Yellow Spring Instrument Co.). Plasma C-peptide, insulin, glucagon, active GLP-1, leptin, adiponectin, and CRP concentrations were measured using enzyme-linked immunosorbent assays (Millipore). Plasma glucose TTRs were determined by using gas chromatography-mass spectrometry (30).

\section{Analyses of adipose tissue samples}

Isolation of $m R N A$ and quantitative PCR. Frozen adipose tissue samples were homogenized in RNAzol Bee (Tel-Test Inc.), and RNA was isolated according to the manufacturer's protocol. Gene expression was determined using quantitative real-time PCR. cDNA was synthesized by using SuperScript VILO (Life Technologies), and cDNA samples were amplified by using SybrGreen reagent and the ABI 7500 thermal cycler (Life Technologies). The expression of each gene (EMR1, CD11B, CCL2, CSF3, IL6, TNFA, IL10, and $L E P$ ) was determined by correcting the threshold crossing $(\mathrm{Ct})$ of each sample to the housekeeping control gene, acidic ribosomal phosphoprotein P0 (36B4), calculated as $2^{-\Delta C t}$. The following primer sequences were used: EMR1, GGAAGGGCACATAAGACCCAG and GGGCACAAGG-
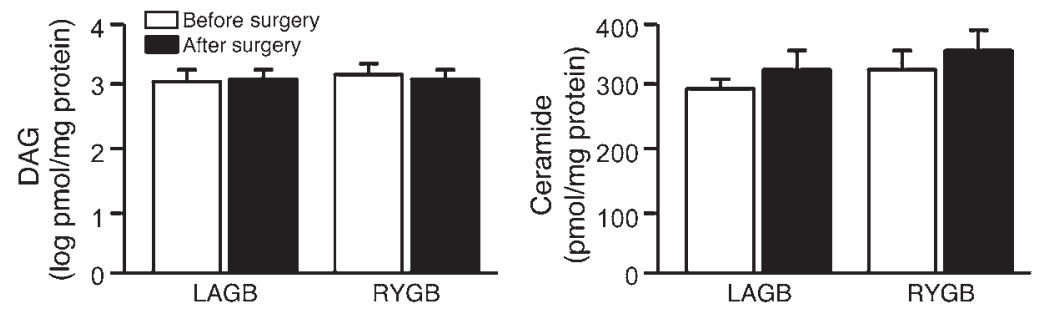

Skeletal muscle DAG and ceramide content before and after $20 \%$ weight loss induced by LAGB $(n=10)$ or RYGB $(n=7)$ surgery. Values are means \pm SEM. 
TACTGTCTCTA; CD11B, CAGCCTTGACCTTATGTCATGG and CCTGTGCTGTAGTCGCACT; CCL2, ATAGCAGCCACCTTCATTCC and GCTTCTTTGGGACACTTGCT; CSF3, GCGGCTTGAGCCAACTCCATA and GAACGCGGTACGACACCTC; IL6, CCTGAACCTTCCAAAGATGG and TGGCTTGTTCCTCACTACTCTC; TNFA, GGAAAGGACACCATGAGCA and CAGAGGGCTCATTAGAGAGAGG; IL10, TCAAGGCGCATGTGAA and GATGTCAAACTCACTC; LEP, GTGGCTTTGGCCCTATCTT and GCATACTGGTGAGGATCTGTTG; 36B4, GTGATGTGCAGCTGATCAAGACT and GATGACCAGCCCAAAGGAGA.

\begin{abstract}
Analyses of skeletal muscle lipid metabolites
Intramyocellular DAG and ceramide concentrations were determined using mass spectrometry (31). Muscle tissue samples were weighed and lipids were extracted as we previously described (32). About $10 \%$ of the lipid extract was used to evaluate ceramide content. Quantitation of ceramide molecular species was performed by using a Thermo triple quadrupole (TSQ) Vantage mass spectrometer and electrospray ionization (ESI) (33). Neutral lipids were extracted from the rest of the lipid extract by hexane and the free hydroxyl group in DAG was protected with 2,4-diflourophenyl isocyanate to quantitate DAG molecular species using an Agilent 1100 LC system connected to an Agilent 6460 TSQ mass spectrometer operated in ESI and positive ion mode (34). The LC system allows separation of DAG species from other neutral lipids and resolution of 1,2-sn-DAG and 1,3-sn-DAG species. Total DAG and ceramide contents were calculated by summation of the individual 1,2-sn-DAG (C22:0, C23:0, C24:0, C25:0, C26:0, C27:0, C28:0, C29:0, C30:1, C30:0, C31:0, C30:0, C31:0, C32:2, C32:1, C32:0, C33:1, C33:0, C34:3, C34:2, C34:1, C34:0, C35:1, C35:0, C36:4, C36:3, C36:2, C36:1, C36:0, C38:5, C38:4, C38:3, C38:2, C40:5) and 1,3-sn-DAG (C22:0, C24:0, C26:0, C28:0, C30:0, C32:1, C32:0, C34:2, C34:1, C34:0, C36:3, C36:2, C36:1, C36:0) species and the individual ceramide (C16:0, C18:1, C18:0, C19:0, C20:0, C22:0, C23:0, C24:2, C24:1, C24:0, C24:1OH, C24:0OH) species.
\end{abstract}

\section{Calculations}

Insulin sensitivity. HOMA-IR was used to provide an index of whole-body insulin resistance (35). Glucose Ra into plasma was calculated by dividing the tracer infusion rate by the average plasma glucose TTR during the last 30 minutes of the basal and insulin infusion periods (36). Glucose disposal rate was equal to endogenous glucose Ra plus the rate of exogenously infused dextrose and glucose tracer. Insulin-stimulated glucose uptake was used as an index of skeletal muscle insulin sensitivity.

$\beta$ Cell function. Minimal model analysis was used to calculate $\Phi_{\text {total }}$, an overall index of postprandial insulin secretion in response to plasma glucose, using plasma C-peptide as a function of glucose concentration (37). The minimal model can also resolve the total ISR into 2 components representing the release of insulin from 2 distinct pools of insulin secretory granules within the $\beta$ cell: a dynamic component that represents the rapid release of a "readily releasable pool," which usually accounts for less than $10 \%$ of total insulin secretory granules, and a static component that represents the slower release of a "reserve pool" of insulin secretory granules (38). The DI, which provides an assessment of insulin secretion in relationship to insulin sensitivity (39), was calculated as the product of $\Phi_{\text {total }}$ and the change in glucose Rd per kg FFM per change in insulin concentration during the hyperinsulinemic-euglycemic clamp procedure.

Metabolic response to the mixed meal. Plasma glucose, insulin, and C-peptide concentration AUCs for 6 hours after initiating meal consumption were calculated using the trapezoid method (40). Total glucose Ra into the systemic circulation during the meal was calculated using Steele's equation for non-steady-state conditions (36). Glucose Ra into the systemic circulation from ingested glucose and from EGP were calculated as previously described (41).

\section{Statistics}

Data were examined for normality according to the Shapiro-Wilks criteria, and non-normally distributed data sets were log-transformed before statistical analyses were performed. A 2-way repeated measures analysis of variance with Tukey's post-hoc procedure was used to compare the effects of RYGB and LAGB surgery on study outcome measures (except for postprandial metabolic outcomes) in the 2 groups. For postprandial metabolic outcomes, mixed-model repeated-measures analysis of variance was used to evaluate differences in weight loss-induced changes between LAGB and RYGB groups. A $P$ value of 0.05 or less was considered statistically significant. All data are presented as means \pm SD unless otherwise indicated.

Based on the interindividual variability of glucose rate of disappearance (Rd) during a hyperinsulinemic-euglycemic clamp procedure in a large cohort of nondiabetic obese subjects we previously studied (42), we estimated that 10 subjects in each group (RYGB and LAGB) would be needed to detect a $40 \%$ difference in insulin-stimulated glucose Rd between the RYGB and the LAGB group with a $\beta$ value of 0.20 (i.e., $80 \%$ power) and an $\alpha$ value of 0.05 . This proposed difference between groups is a conservative estimate based on the results from the only 2 previous studies comparing the effect of gastric restriction versus RYGB procedures on insulin sensitivity using the hyperinsulinemic clamp procedure $(43,44)$. In both studies, insulin-stimulated glucose disposal was more than $200 \%$ greater after RYGB than after restrictive surgical procedures.

\section{Study approval}

This study was approved by the Human Research Protection Office at Washington University School of Medicine. All study subjects provided written informed consent before screening and participation in the study.

\section{Acknowledgments}

The authors thank Courtney Tiemann for help with subject recruitment and dietary counseling; Hua Cheng, Freida Custodio, Jennifer Shew, Ioana Gruchevska, and Terri Pietka for technical assistance; Faidon Magkos and Kenneth Schechtman for statistical support; the staff of the Clinical Research Unit for their help in performing the studies; and the study subjects for their participation. This study was supported by NIH grants DK 37948 , DK 56341 (Nutrition Obesity Research Center), UL1 RR024992 (Clinical and Translational Science Award), and RR-00954 (Biomedical Mass Spectrometry Resource); by a grant from Ethicon Endo-Surgery; and by the Atkins Foundation Philanthropic Trust.

Received for publication May 21, 2012, and accepted in revised form September 6, 2012.

Address correspondence to: Samuel Klein, Center for Human Nutrition, Washington University School of Medicine, 660 South Euclid Avenue, Campus Box 8031, St. Louis, Missouri 63110, USA. Phone: 314.362.8708; Fax: 314.362.8230; E-mail: sklein@dom.wustl.edu.
1. DeFronzo RA. Pathogenesis of type 2 diabetes mellitus. Med Clin North Am. 2004;88(4):787-835, ix.

2. Niskanen L, Uusitupa M, Sarlund H, Siitonen O,

Paljarvi L, Laakso M. The effects of weight loss on insulin sensitivity, skeletal muscle composition and capillary density in obese non-diabetic subjects. Int J Obes Relat Metab Disord. 1996;20(2):154-160.

3. Gregor MF, et al. Endoplasmic reticulum stress is reduced in tissues of obese subjects after weight loss. Diabetes. 2009;58(3):693-700.
4. Villareal DT, Banks MR, Patterson BW, Polonsky KS, Klein S. Weight loss therapy improves pancreatic endocrine function in obese older adults. Obesity (Silver Spring). 2008;16(6):1349-1354.

5. Guldstrand M, Ahren B, Adamson U. Improved 
beta-cell function after standardized weight reduction in severely obese subjects. Am J Physiol Endocrinol Metab. 2003;284(3):E557-E565.

6. Buchwald $\mathrm{H}$, et al. Weight and type 2 diabetes after bariatric surgery: systematic review and meta-analysis. Am J Med. 2009;122(3):248-256.e5.

7. Chawla A, Nguyen KD, Goh YP. Macrophagemediated inflammation in metabolic disease. Nat Rev Immunol. 2011;11(11):738-749.

8. Eckardt K, Taube A, Eckel J. Obesity-associated insulin resistance in skeletal muscle: role of lipid accumulation and physical inactivity. Rev Endocr Metab Disord. 2011;12(3):163-172.

9. Jornayvaz FR, Samuel VT, Shulman GI. The role of muscle insulin resistance in the pathogenesis of atherogenic dyslipidemia and nonalcoholic fatty liver disease associated with the metabolic syndrome. Annu Rev Nutr. 2010;30:273-290.

10. Lima MM, et al. Acute effect of roux-en-y gastric bypass on whole-body insulin sensitivity: a study with the euglycemic-hyperinsulinemic clamp. JClin Endocrinol Metab. 2010;95(8):3871-3875.

11. Campos GM, et al. Improvement in peripheral glucose uptake after gastric bypass surgery is observed only after substantial weight loss has occurred and correlates with the magnitude of weight lost. J Gastrointest Surg. 2010;14(1):15-23.

12. Petersen KF, Dufour S, Befroy D, Lehrke M, Hendler RE, Shulman GI. Reversal of nonalcoholic hepatic steatosis, hepatic insulin resistance, and hyperglycemia by moderate weight reduction in patients with type 2 diabetes. Diabetes. 2005;54(3):603-608.

13. Sato F, et al. Effects of diet-induced moderate weight reduction on intrahepatic and intramyocellular triglycerides and glucose metabolism in obese subjects. J Clin Endocrinol Metab. 2007;92(8):3326-3329.

14. Elder DA, Prigeon RL, Wadwa RP, Dolan LM, D'Alessio DA. Beta-cell function, insulin sensitivity, and glucose tolerance in obese diabetic and nondiabetic adolescents and young adults. J Clin Endocrinol Metab. 2006;91(1):185-191.

15. Wang G, et al. Accelerated gastric emptying but no carbohydrate malabsorption 1 year after gastric bypass surgery (GBP). Obes Surg. 2012;22(8):1263-1267.

16 . Bose $\mathrm{M}$, et al. Weight loss and incretin responsiveness improve glucose control independently after gastric bypass surgery. J Diabetes. 2012;2(1):47-55.

17. Salehi M, Prigeon RL, D’Alessio DA. Gastric bypass surgery enhances glucagon-like peptide 1-stimulated postprandial insulin secretion in humans. Diabetes. 2011;60(9):2308-2314.
18. Temelkova-Kurktschiev TS, et al. Postchallenge plasma glucose and glycemic spikes are more strongly associated with atherosclerosis than fasting glucose or HbA1c level. Diabetes Care. 2000;23(12):1830-1834.

19. Anan F, et al. Postchallenge plasma glucose and glycemic spikes are associated with pulse pressure in patients with impaired glucose tolerance and essential hypertension. Hypertens Res. 2008;31(8):1565-1571.

20. Hu Y, Liu W, Huang R, Zhang X. Postchallenge plasma glucose excursions, carotid intima-media thickness, and risk factors for atherosclerosis in Chinese population with type 2 diabetes. Atherosclerosis. 2010;210(1):302-306.

21. Esposito K, et al. Post-meal glucose peaks at home associate with carotid intima-media thickness in type 2 diabetes. J Clin Endocrinol Metab. 2008; 93(4):1345-1350.

22. Samuel VT, Shulman GI. Mechanisms for insulin resistance: common threads and missing links. Cell. 2012;148(5):852-871.

23. Bikman BT, Summers SA. Ceramides as modulators of cellular and whole-body metabolism. J Clin Invest. 2011;121(11):4222-4230.

24. Samuel VT, Petersen KF, Shulman GI. Lipidinduced insulin resistance: unravelling the mechanism. Lancet. 2010;375(9733):2267-2277.

25. Schauer PR, et al. Effect of laparoscopic Roux-en Y gastric bypass on type 2 diabetes mellitus. Ann Surg. 2003;238(4):467-484

26. Frimel TN, Deivanayagam S, Bashir A, O'Connor $\mathrm{R}$, Klein S. Assessment of intrahepatic triglyceride content using magnetic resonance spectroscopy. J Cardiometab Syndr. 2007;2(2):136-138.

27. Vitola BE, et al. Weight loss reduces liver fat and improves hepatic and skeletal muscle insulin sensitivity in obese adolescents. Obesity (Silver Spring). 2009; 17(9):1744-1748.

28. Varela JE. Bariatric surgery: a cure for diabetes? Curr Opin Clin Nutr Metab Care. 2011;14(4):396-401.

29. Varela JE, Hinojosa MW, Nguyen NT. Perioperative outcomes of bariatric surgery in adolescents compared with adults at academic medical centers. Surg Obes Relat Dis. 2007;3(5):537-540.

30. Fabbrini E, et al. Intrahepatic fat, not visceral fat, is linked with metabolic complications of obesity. Proc Natl Acad Sci U S A. 2009;106(36):15430-15435.

31. Magkos F, et al. Intrahepatic diacylglycerol content is associated with hepatic insulin resistance in obese subjects. Gastroenterology. 2012;142(7):1444-1446.e2.

32. Li YL, Su X, Stahl PD, Gross ML. Quantification of diacylglycerol molecular species in biological samples by electrospray ionization mass spectrometry after one-step derivatization. Anal Chem. 2007;79(4):1569-1574.

33. Han X. Characterization and direct quantitation of ceramide molecular species from lipid extracts of biological samples by electrospray ionization tandem mass spectrometry. Anal Biochem. 2002; 302(2):199-212.

34. Leiker TJ, Barkley RM, Murphy RC. Analysis of diacylglycerol molecular species in cellular lipid extracts by normal-phase LC-electrospray mass spectrometry. Int J Mass Spectrom. 2011;305(2-3):103-109.

35. Matthews DR, Hosker JP, Rudenski AS, Naylor BA, Treacher DF, Turner RC. Homeostasis model assessment: insulin resistance and beta-cell function from fasting plasma glucose and insulin concentrations in man. Diabetologia. 1985;28(7):412-419.

36. Steele R. Influences of glucose loading and of injected insulin on hepatic glucose output. Ann NY Acad Sci. 1959;82:420-430.

37. Breda E, Toffolo G, Polonsky KS, Cobelli C. Insulin release in impaired glucose tolerance: oral minimal model predicts normal sensitivity to glucose but defective response times. Diabetes. 2002; 51(suppl 1):S227-S233.

38. Seino S, Shibasaki T, Minami K. Dynamics of insulin secretion and the clinical implications for obesity and diabetes. J Clin Invest. 2011;121(6):2118-2125.

39. Bergman RN, Ader M, Huecking K, Van Citters G. Accurate assessment of $\beta$-cell function: the hyperbolic correction. Diabetes. 2002;51(suppl 1):S212-S220.

40. Tai MM. A mathematical model for the determination of total area under glucose tolerance and other metabolic curves. Diabetes Care. 1994;17(2):152-154.

41. Gastaldelli A, et al. Effect of pioglitazone on the metabolic and hormonal response to a mixed meal in type II diabetes. Clin Pharmacol Ther. 2007; 81(2):205-212.

42. Conte C, Fabbrini E, Kars M, Mittendorfer B, Patterson BW, Klein S. Multiorgan insulin sensitivity in lean and obese subjects. Diabetes Care. 2012;35(6):1316-1321.

43. Kashyap SR, et al. Acute effects of gastric bypass versus gastric restrictive surgery on beta-cell function and insulinotropic hormones in severely obese patients with type 2 diabetes. Int J Obes (Lond). 2010;34(3):462-471.

44. Abbatini F, et al. Long-term effects of laparoscopic sleeve gastrectomy, gastric bypass, and adjustable gastric banding on type 2 diabetes. Surg Endosc. 2010;24(5):1005-1010. 